Trinity University

Digital Commons @ Trinity

Engineering Faculty Research

Engineering Science Department

2011

\title{
Reduction of Vibration Transmission in String Trimmers
}

Paurakh Rajbhandary

Trinity University

Jack Leifer

Trinity University, jleifer@trinity.edu

Bryan J. Weems

Trinity University

Follow this and additional works at: https://digitalcommons.trinity.edu/engine_faculty

Part of the Engineering Commons

\section{Repository Citation}

T. Proulx (ed.), Rotating Machinery, Structural Health Monitoring, Shock and Vibration, Volume 5, Conference Proceedings of the Society for Experimental Mechanics Series 8, 2011, pp. 129 - 138.

This Post-Print is brought to you for free and open access by the Engineering Science Department at Digital Commons @ Trinity. It has been accepted for inclusion in Engineering Faculty Research by an authorized administrator of Digital Commons @ Trinity. For more information, please contact jcostanz@trinity.edu. 


\title{
Reduction of Vibration Transmission in String Trimmers
}

\author{
Paurakh Rajbhandary, Undergraduate Research Assistant \\ Jack Leifer, PhD, PE, Associate Professor of Engineering Science \\ Bryan J. Weems, Undergraduate Research Assistant \\ Department of Engineering Science \\ Trinity University, One Trinity Place, San Antonio, TX 78212
}

\begin{abstract}
It has been well documented that over long periods of time, people who regularly operate hand tools powered by small internal-combustion engines can become affected by a debilitating set of clinically irreversible effects, collectively referred to as hand-arm vibration syndrome (HAVS). Although HAVS cannot be cured, the onset of the disorder can be delayed or, in fact, prevented, by restricting either the duration of the exposure, and/or the magnitude of the vibration transmitted from the tool to an operator's hands and arms (per ANSI and similar standards). In this paper, we've evaluated an approach for reducing the magnitude of transmitted vibration at the engine-side grip of one commercial- and one consumer-grade string trimmer. The ultimate goal of this work is to develop a set of simple, passive retrofits for these tools that will reduce the likelihood of HAVS in full-time grounds-maintenance workers. The idea for this work stems from a stated need of Goodwill Industries of San Antonio, our NPA (non-profit agency) collaborator over the past several years. Based on this preliminary work, we've found that grips incorporating half-inch rubber studs placed at areal densities of either $25 / \mathrm{in}^{2}$ or $12 / \mathrm{in}^{2}$ significantly reduce the RMS vibration transmitted to the hand and arm at all frequencies (including the low frequency components known to be most harmful).
\end{abstract}

\section{Introduction}

Hand-arm Vibration Syndrome (HAVS) is an irreversible condition that affects individuals who are exposed to certain levels of vibration from equipment powered by internal combustion engines on a long-term basis. The recent survey by Pelmear and Wasserman states that HAVS may arise after long-term exposure to all types and sizes of vibrating, hand-operated tools, including jack hammers, impact wrenches, orthodontic grinders, floor polishers, chain saws, and string trimmers. [1] Symptoms of HAVS include blanching of the fingers, tingling and numbness, and are generally more prevalent in cold weather. While HAVS cannot generally be cured, its onset and severity can be controlled through the combined approach of reducing both the magnitude and/or the duration of the exposure to the vibrations generated by the tools. Such an approach has been well documented by a variety of standards, including ISO 5349-1 and ANSI S2.70. [2,3] Therefore, for workers whose jobs require them to operate such tools for many hours per day, the only practical method of limiting the onset of HAVS is to reduce the amplitude of the vibration transmitted through the tools into their hands and arms.

The objective of this on-going study is to quantify the effect that various padding materials have on reducing the magnitude of transmitted vibration in string trimmers, with the ultimate goal of retrofitting these types of tools to decrease the likelihood of HAVS in full-time grounds-maintenance workers. Referring to the photograph shown in Fig. 1, string trimmers are generally held in two locations so they can be maneuvered as they are used. The handgrip located at the top of the shaft, adjacent to the engine, is supported by the same hand that also actuates the throttle control. The loop-shaped handle is located further down the shaft, and allows the orientation of the string-trimmer to be changed by simply changing grip position on the handle. In order to passively reduce transmitted vibration, padding materials can be introduced in a variety of ways: (1) as a replacement for the manufacturer's handgrip (held by the operator's hand below the engine); (2) as a wrap applied over the loop- 


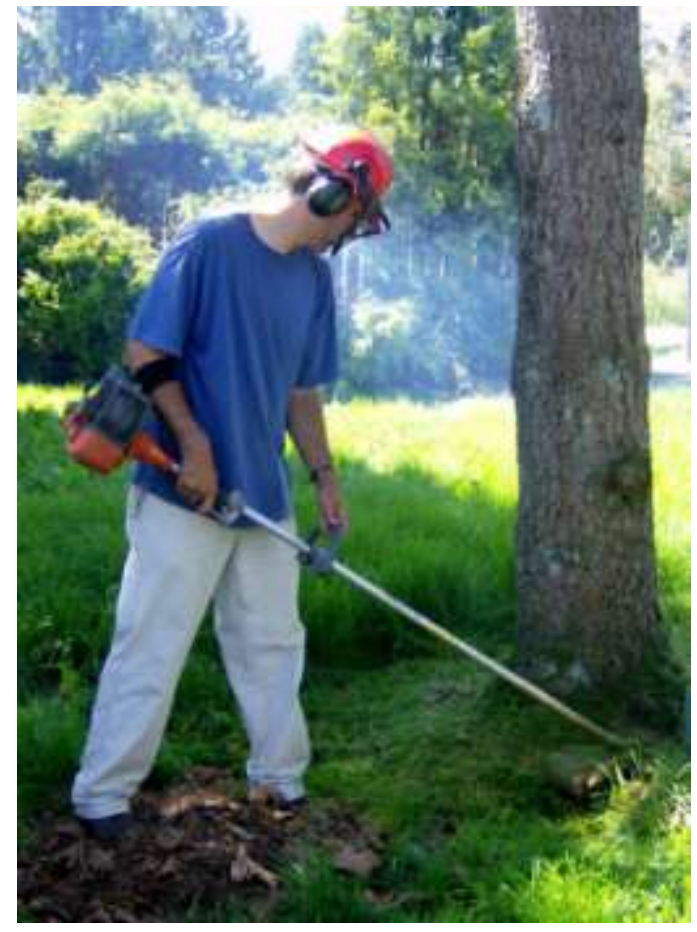

Fig. 1 A typical string trimmer is supported by a grip adjacent to the engine (left) and a loopshaped handle (right) located further down the shaft [4]

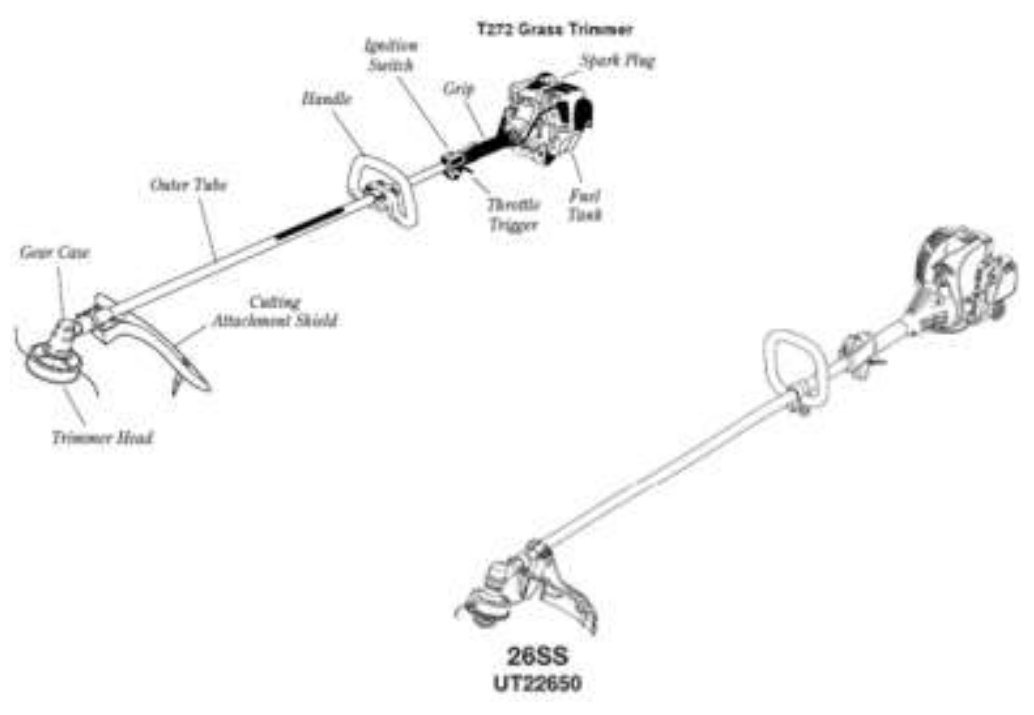

Fig. 2 Schematic of Shindaiwa (top) and Homelite (bottom) string trimmers used in this study. [5,6]

ase of elastomeric materials to reduce ials to reduce transmitted vibration must be considered carefully, as the increased perception of user comfort offered by padded handles and grips does not always indicate a decreased risk for HAVS. As described by ISO 5349-1 [2], daily vibration exposure, $A(8)$, is a parameter that considers episode duration along with a weighted RMS acceleration magnitude $\left(a_{h w}\right)$ as follows: intended for the home (consumer) market. Its two-stroke engine is slightly smaller than that of the Shindaiwa. It is apparent from the schematics that both string trimmers have essentially the same layout - each one has an engine that is mounted atop a straight shaft, and the engine on each unit is started with a typical pull-starter. Engine speed is controlled by a throttle mounted on the shaft immediately adjacent to a padded grip, and a loop-type handle that extends normal to the string trimmer is mounted on each shaft about a foot below the grip/throttle combination. In both cases, the handle is mounted using a sleeve fit, and tightness against the shaft is controlled by one (Homelite) or four (Shindaiwa) bolts.

(c) Passive Damping Materials. The

shaped handle; and (3) as a wrap installed between the loop-shaped handle and the shaft of the tool. In this study, we concentrated on evaluating materials that could replace the manufacturer's engine-side handgrip.

\section{Materials and Methods}

(a) Accelerometers and DAQ Boards. In order to measure all three components of generated vibration simultaneously, two similar Piezotronics U339B01 triaxial ICP accelerometers were used for various experiments in this study. The accelerometers each had a resolution of $0.002 \mathrm{~g}$ between $1 \mathrm{~Hz}$ and $2 \mathrm{kHz}$, and nominal sensitivities in the $x, y$ and $z$ directions of $90.9 \mathrm{mV} / \mathrm{g}, 89.1 \mathrm{mV} / \mathrm{g}$, and $90.9 \mathrm{mV} / \mathrm{g}$ (SN 2988) and $99.1 \mathrm{mV} / \mathrm{g}, 93.9 \mathrm{mV} / \mathrm{g}$, and $71.6 \mathrm{mV} / \mathrm{g}$ (SN 3198). Both accelerometers were interfaced to an lotech Wavebook516 16-bit Data Acquisition System equipped with an eight-channel WBK14 expansion module specifically designed for ICP accelerometers.

(b) String Trimmers. While every engine and chassis combination produces a unique vibration signature, the general effects of the vibration mitigation approaches evaluated herein are likely applicable to a whole range of string trimmers and other similar hand-held power tools. In order to test this hypothesis, measurements were taken on two different string trimmers. (1) The Shindaiwa T272 (Fig. 2 ) is intended for the commercial market. Equipped with a $1.4 \mathrm{hp} 2$-stroke engine, the dry weight of the unit is approximately 14.7 pounds. (2) The Homelite 26SS (Fig. 2 ) is lighter (weighs only $10 \mathrm{lbs}$ ), and is (n) 


$$
a_{h v}=\sqrt{a_{h w x}^{2}+a_{h w y}^{2}+a_{h w z}^{2}}
$$

and

$$
A(8)=\sqrt{\frac{1}{8} \sum_{i=1}^{n} a_{h v i}^{2} T_{i}}
$$

where $a_{h w}$ in Eq. 1 simply represents the magnitude of the RMS values of the weighted acceleration components calculated in each direction (with respect to a basicentric coordinate system), and $A(8)$ in Eq. 2 represents the RMS value of each individual exposure (of duration $T_{i}$ ) normalized to an 8-hour workday. Note that ISO 5349-1 has established a frequency-weighting factor scheme that considers the relative effect that each frequency component has on hand injury. It has been found that low frequency components are most injurious, therefore the highest relative weightings (between 0.873 and 1.00) are concentrated between frequency components centered from $8 \mathrm{~Hz}-16 \mathrm{~Hz}$.

Researchers such as Ko have found that elastomeric wraps can reduce $A(8)$ by nearly $50 \%$. [7] However, others sources warn that wraps may not be effective at the vibration frequencies that contribute most to the daily exposure levels (i.e. at low frequencies), and may in fact cause $A(8)$ vibration exposure to increase. [8] Rather than evaluate flat elastomeric materials (e.g. santoprene, closed-cell neoprene) that have been studied previously, we chose to test the effectiveness of the studded rubber mats shown in Fig. 3. The base of each mat was approximately $3 / 32$ inch thick, and the studs were distributed uniformly at an aerial density of $25 \mathrm{studs} / \mathrm{in}^{2}$ over the mat surface in its as-purchased condition. Although the stud diameter $(0.125 \mathrm{inch})$ was the same on both

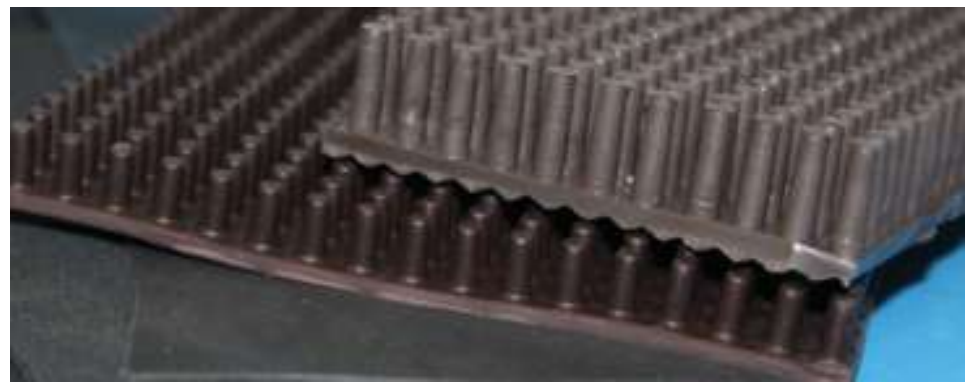

Fig. 3 Studded rubber mats used as wraps in the attenuation tests. The studs on the bottom mat were 0.25 in long, the studs the top mat were 0.50 inch long. mats pictured, stud length was 0.25 inch and 0.50 inch, respectively, on the lower and upper mats shown in Fig. 3. The mats with the 0.5 inch studs were sourced from two different suppliers, and were referred to respectively (based on their physical behavior) as "stiff and compliant" in the test section, below. Studded mats were chosen because it was proposed that this particular material configuration would provide both damping and compliance characteristics that would reduce vibration transmission in both the radial (along the studs) and transverse directions. This hypothesis is based in part on the work of Andersson, who implemented a "vibration damping handle" consisting of both damping and compliant elements that effectively reduced measured vibration between approximately 5 - 1500 Hz. [9]

\section{Experimental}

In all cases, a single accelerometer was used and aligned with a basicentric coordinate system (as suggested by ISO 5349-1) defined by the shaft axis, radius (line directed from the shaft axis to its surface), and tangent (normal to both the radial and axial components). For each experiment, the accelerometer was attached to the string trimmer using cyanoacrylate adhesive (superglue) and standard glue-mounting studs that were provided with the accelerometers. In order to ensure a flat, stable mounting surface for the accelerometer glue stud, a flat was milled into the surface of the elastomeric material under evaluation (Fig. 4a). In cases where milling a flat into a curved surface was not practical (e.g. on the bare shaft of the string trimmer), a custom aluminum adapter was used to couple between the flat glue stud and curved surface (Fig 4b).

Early in the testing sequence, it was noted that both the magnitude and frequency content of the measurements were dependent on a variety of parameters including engine speed, human factors such as hand pressure and position, and string trimmer orientation. In order to reduce the effect of these variables, initial measurements were performed on a string trimmer suspended horizontally in a "free-free" orientation approximated by two 
bungee cords separated by approximately 35 inches (Fig. 5). In order to ensure that the bungee cords did not move during the testing sequence, small pieces of masking tape were used to lightly secure their position against the string trimmer's shaft.

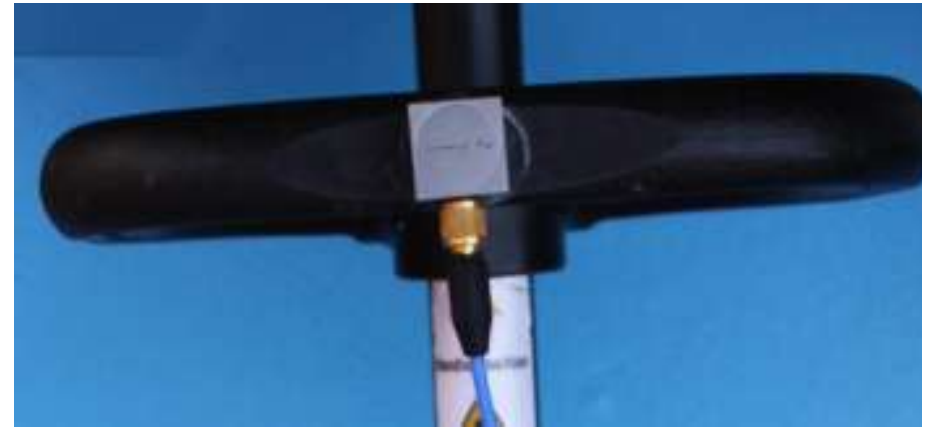

Fig. 4a Accelerometer mounted on handle, aligned with a basicentric coordinate system. Note the flat milled into the surface of the handle facilitates a secure coupling

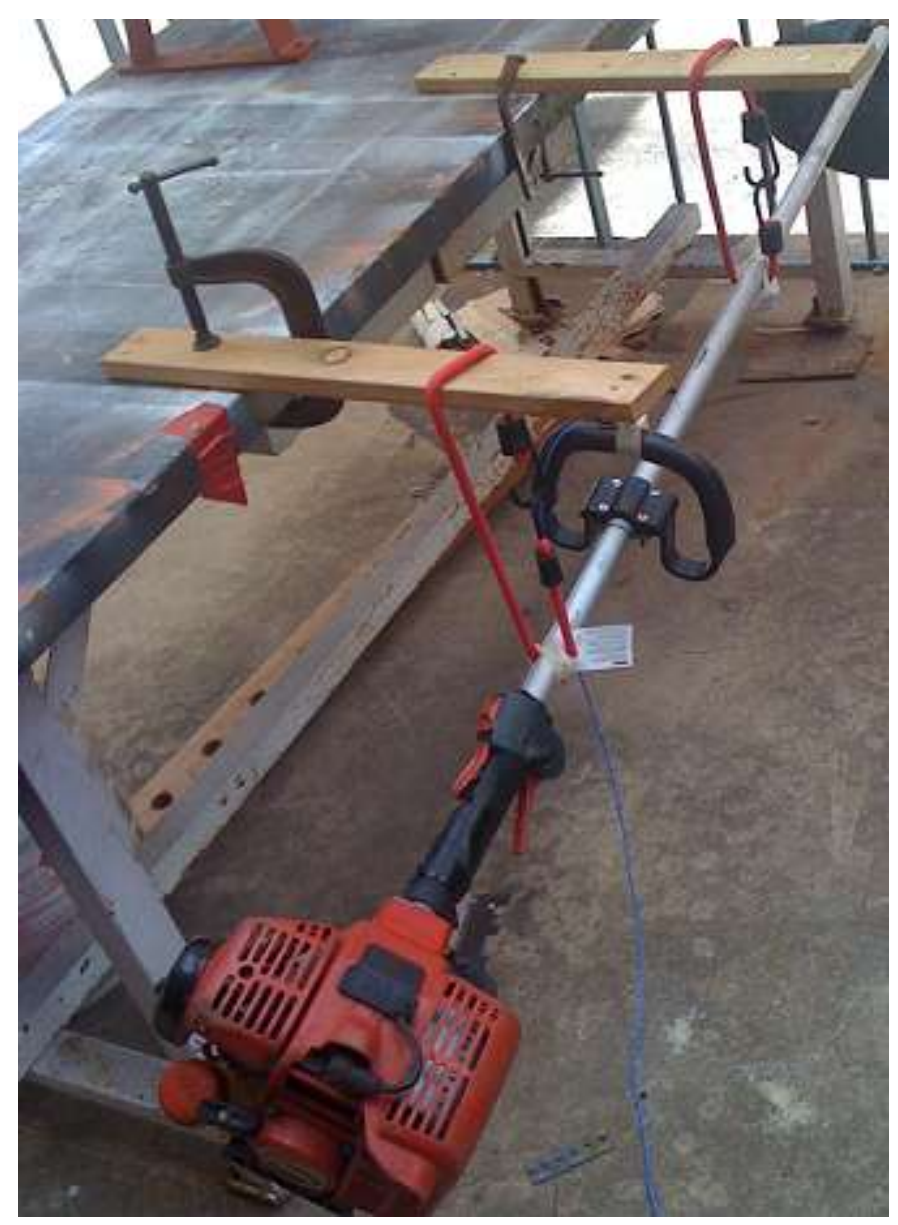

Fig. 5 Free-free suspension approach for string trimmer baseline test. The Shindaiwa trimmer is shown here.

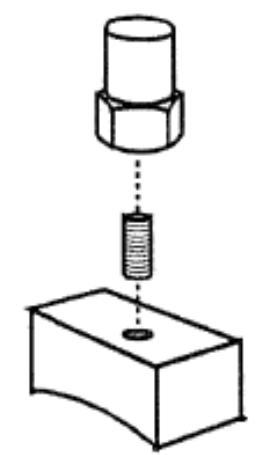

Fig. 4b Schematic representation of an adapter that allows a flat accelerometer to be mounted to a contoured surface (adapted from ISO 5439-2) [2]

(a) Baseline Test. To obtain the baseline frequency content in each of the basicentric coordinate directions, the loop handle was removed from the each string trimmer, and the accelerometer (SN 2988) was installed in its place. This was done by gluing an adapter (Fig. $4 b)$ to the shaft, and attaching the accelerometer to the adapter using the threaded stud. This accelerometer was located exactly 4 inches below the lower end of the manufacturer's grip. Surface acceleration components were measured at both an unthrottled (idle) and throttled condition. Note that to obtain a throttled measurement, the string trimmer throttle was carefully squeezed by hand without disturbing the hanging free-free support. Acceleration components were measured at this point using the method provided for triaxial measurement in the ISO 5349-2 standard. [2] For both the throttled and unthrottled conditions, data were taken for 5.0 seconds at a sampling frequency of $5000 \mathrm{~Hz}$. Conversion to the frequency domain was accomplished within MatLAB using a fast-fourier transformation algorithm, and typical spectra obtained for each accelerometer channel are shown in Fig. 6 (for the Homelite trimmer only). Note that most of the content in each coordinate direction is concentrated below $500 \mathrm{~Hz}$; furthermore, the vibration amplitude for the throttled condition increased by about an order of magnitude over that obtained for the unthrottled condition. The data presented in Fig. 6 are a typical example of the measurements obtained, and clearly show that string trimmers generate significant vibrations in all three of the basicentric coordinate directions. 


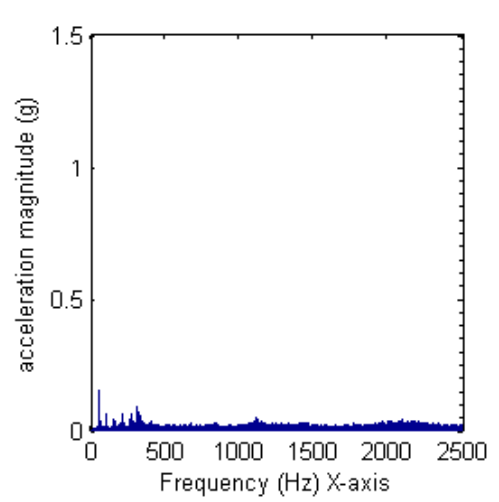

(a) Unweighted, Unthrottled
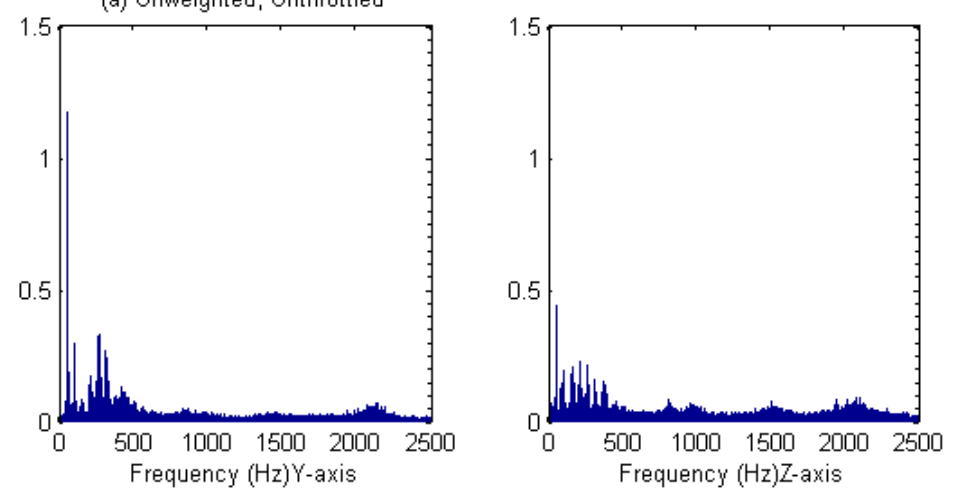

(b) Unweighted, Throttled
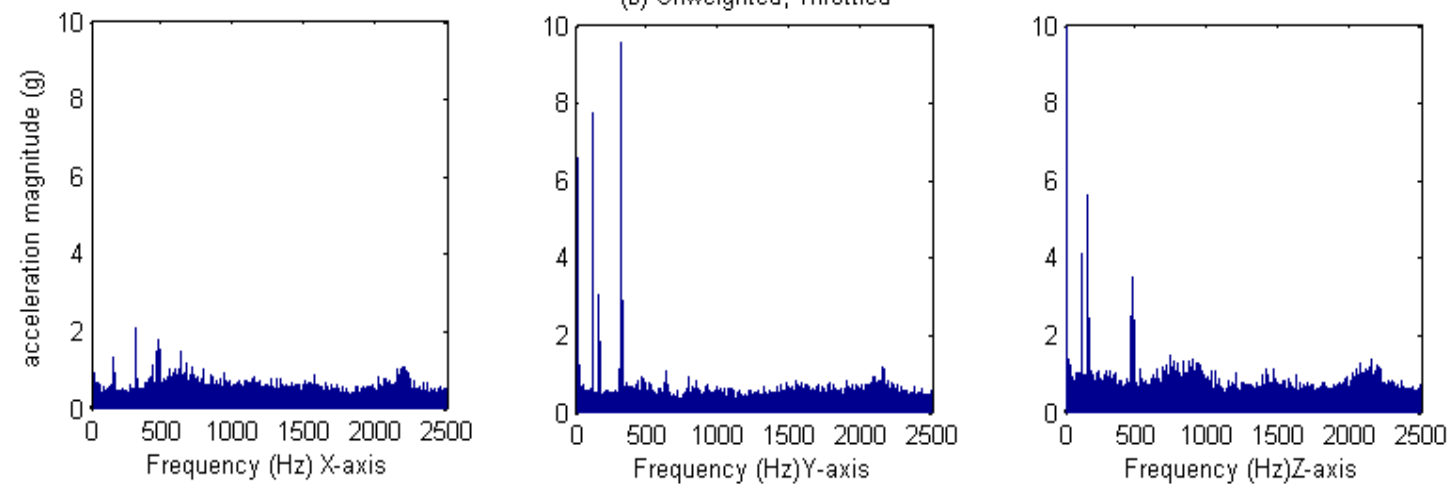

Fig. 6 Baseline frequency domain plots of acceleration components taken by the fixed accelerometer (SN 2988) for the (a) unthrottled and (b) throttled conditions using the Homelite string trimmer

Since Eq. 2 (above) uses acceleration magnitude to calculate $A(8)$; the remainder of the measurements presented herein will report the RMS magnitudes of both the unweighted and weighted acceleration signals rather than the component data.

(b) Grip Test. As mentioned previously, a string trimmer is typically supported in two locations: at the loop handle (held by the operator's left hand in Fig. 1), and at the padded area of the shaft (grip; held by the operator's right hand in Fig.1). At the grip location, the shaft of the string trimmer is covered with a soft padded material that differs from model to model. The function of the padding is to both reduce the vibrations transmitted to the hand and arm of the operator, and to also protect the thin throttle cable that controls engine speed. The padded grip

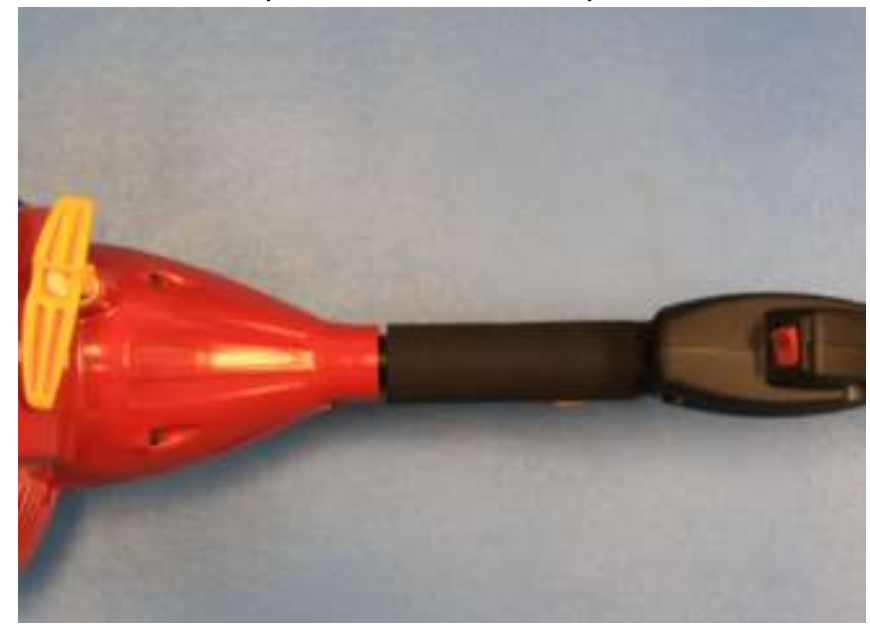

Fig. 7 The grip area of the Homelite string trimmer area on the Shindaiwa is located between the throttle and the engine (Fig. 5); the material used for the grip on the Homelite string trimmer, pictured in Fig. 7, is somewhat thicker. In order to determine whether the magnitude of the transmitted vibration at the grip could be reduced significantly via passive means, the studded mats shown in Fig. 3 were used to fabricate a variety of grips.

For each of the tests performed, the accelerometer (SN 3198) was attached directly to the shaft or the padding (using cyanoacrylate) so that it was positioned directly under the operator's right hand, and aligned with the basicentric coordinate system defined by the shaft of the string trimmer. To ensure that the influence of the hand-arm coupling 
was included in the measurement, the operator gripped the accelerometer and the shaft simultaneously with his or her right hand, in an adaptation of the "moulded hand-held adaptor" approach discussed in the ISO 5349-2 standard. [2] The operator's left hand held the loop handle, allowing the string trimmer to be supported during testing in its typical operating position (although no grass was cut during the tests).

For the two trimmers evaluated, each of the test grips was installed in turn after the manufacturers' grip was removed. The studded material used for each test grip was measured and cut to size (using the original manufacturer's grip as a template), and glued directly to the shaft using cyanoacrylate (Fig. 8, left).
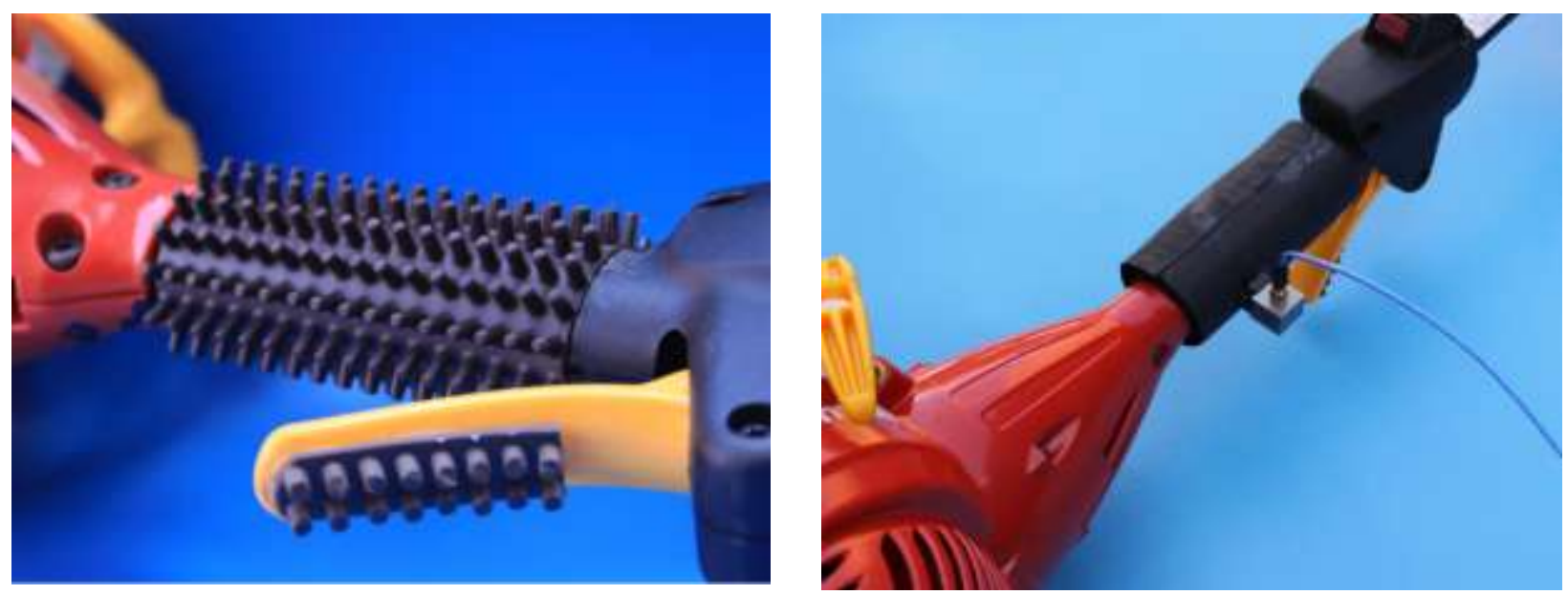

Fig. 8 Quarter-inch studded material is glued directly to the shaft of the Homelite trimmer (left), and then covered with a very thin layer of rubber to facilitate the accelerometer (right). To increase operator comfort, studded material was also installed on the throttle actuator.

\begin{tabular}{|c|c|c|}
\hline Test & Material & Comments \\
\hline 1 & Bare Shaft & $\begin{array}{l}\text { Used as baseline } \\
\text { measurement }\end{array}$ \\
\hline 2 & $\begin{array}{l}\text { Manufacturer's } \\
\text { Grip }\end{array}$ & - \\
\hline 3 & $0.5 "$ studded & Stiff material \\
\hline 4 & $0.5 "$ studded & $\begin{array}{l}\text { Stiff material - half } \\
\text { density (every other stud } \\
\text { removed) }\end{array}$ \\
\hline 5 & $0.25 "$ studded & - \\
\hline 6 & $0.5 "$ studded & Compliant material \\
\hline 7 & $0.5 "$ studded & $\begin{array}{l}\text { Compliant material - } \\
\text { half density }\end{array}$ \\
\hline
\end{tabular}

To ensure comfort (and to provide an appropriate surface for accelerometer mounting), a thin layer of sheet rubber was glued to the top of the studs (Fig. 8, right). For each grip material tested, ten measurements were performed using each string trimmer (five throttled and five unthrottled). A summary of the grip conditions tested is contained in Table 1, and results are presented in Figs. 9-12. For each of the tests performed, the measurement period was set to 20 seconds and a sampling frequency of $5000 \mathrm{~Hz}$ was used. In all cases, the error bars shown represent $+/-1$ standard deviation over the five samples taken for each measurement.

The following general observations can be made based on the data shown in Figs. 9-12:

- The Homelite (consumer model) string trimmer generally transmits more vibration to an operator's hand and arm than the Shindaiwa (commercial) string trimmer.

- When gripped as shown in Fig. 1, the surface accelerations generated by a throttled string trimmer increase by about an order of magnitude over those generated by a string trimmer operating in an idled (unthrottled) condition. This result conforms to that obtained for the frequency-domain data shown in Figs. $6 \mathrm{a}$ and $6 \mathrm{~b}$ for the Homelite string trimmer suspended in a free-free condition.

- The Homelite manufacturer's grip (condition \#2) provides some protection against transmitted vibration when compared with the bare shaft (condition \#1) for both the throttled and unthrottled conditions. 


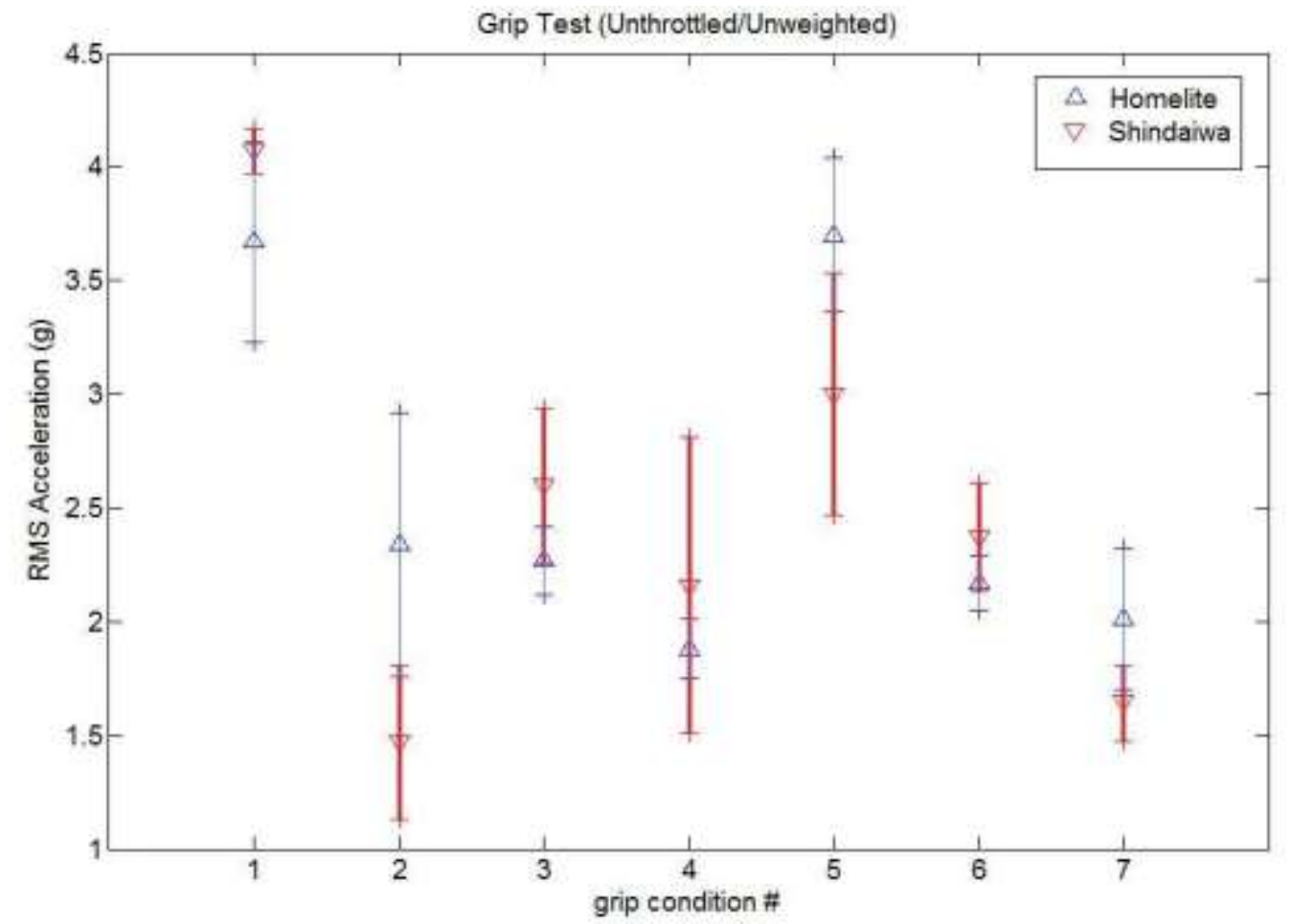

Fig. 9 Performance of various grips under unthrottled engine conditions (unweighted)

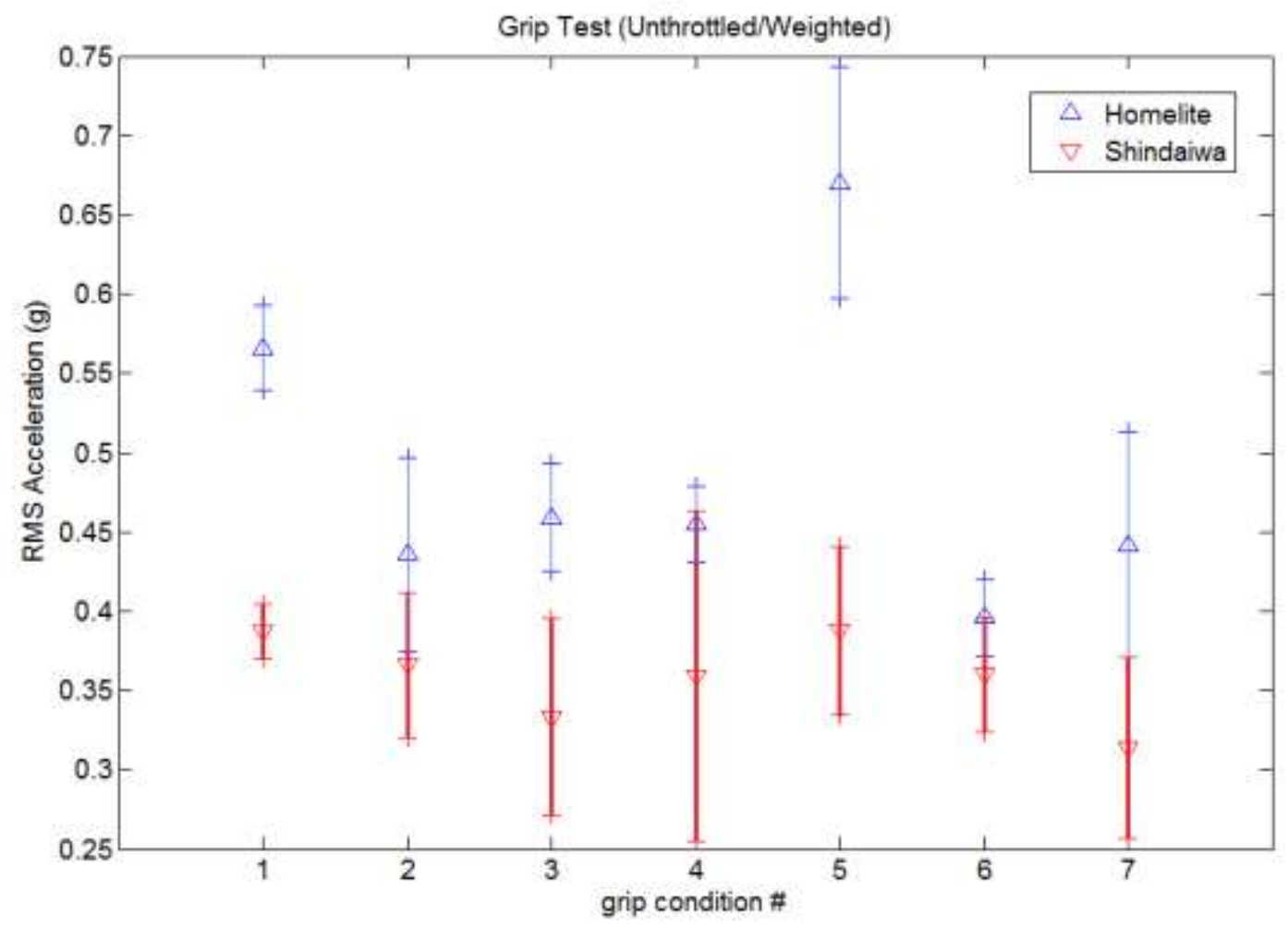

Fig. 10 Performance of various grips under unthrottled engine conditions (weighted) 


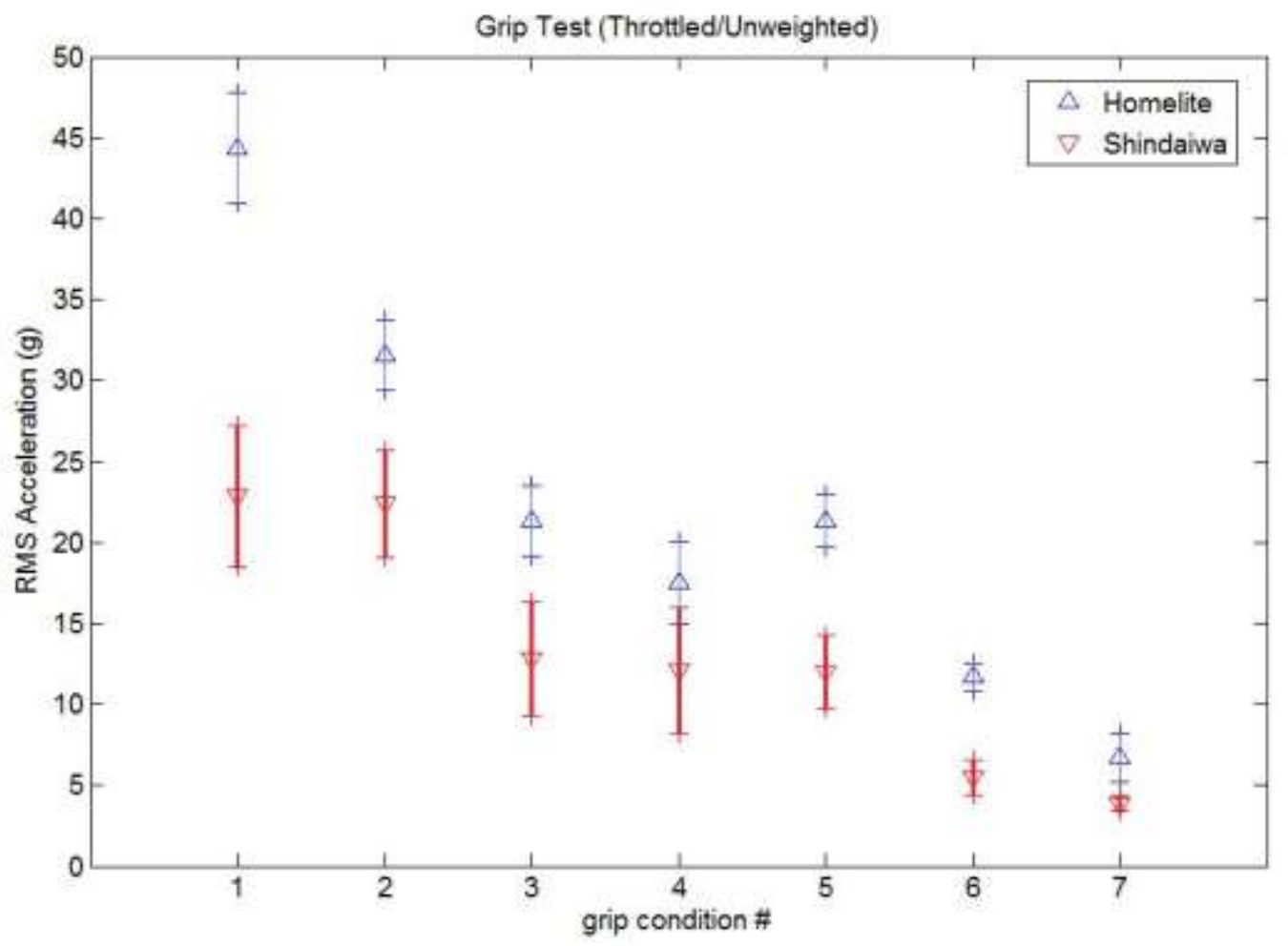

Fig. 11 Performance of various grips under throttled engine conditions (unweighted)

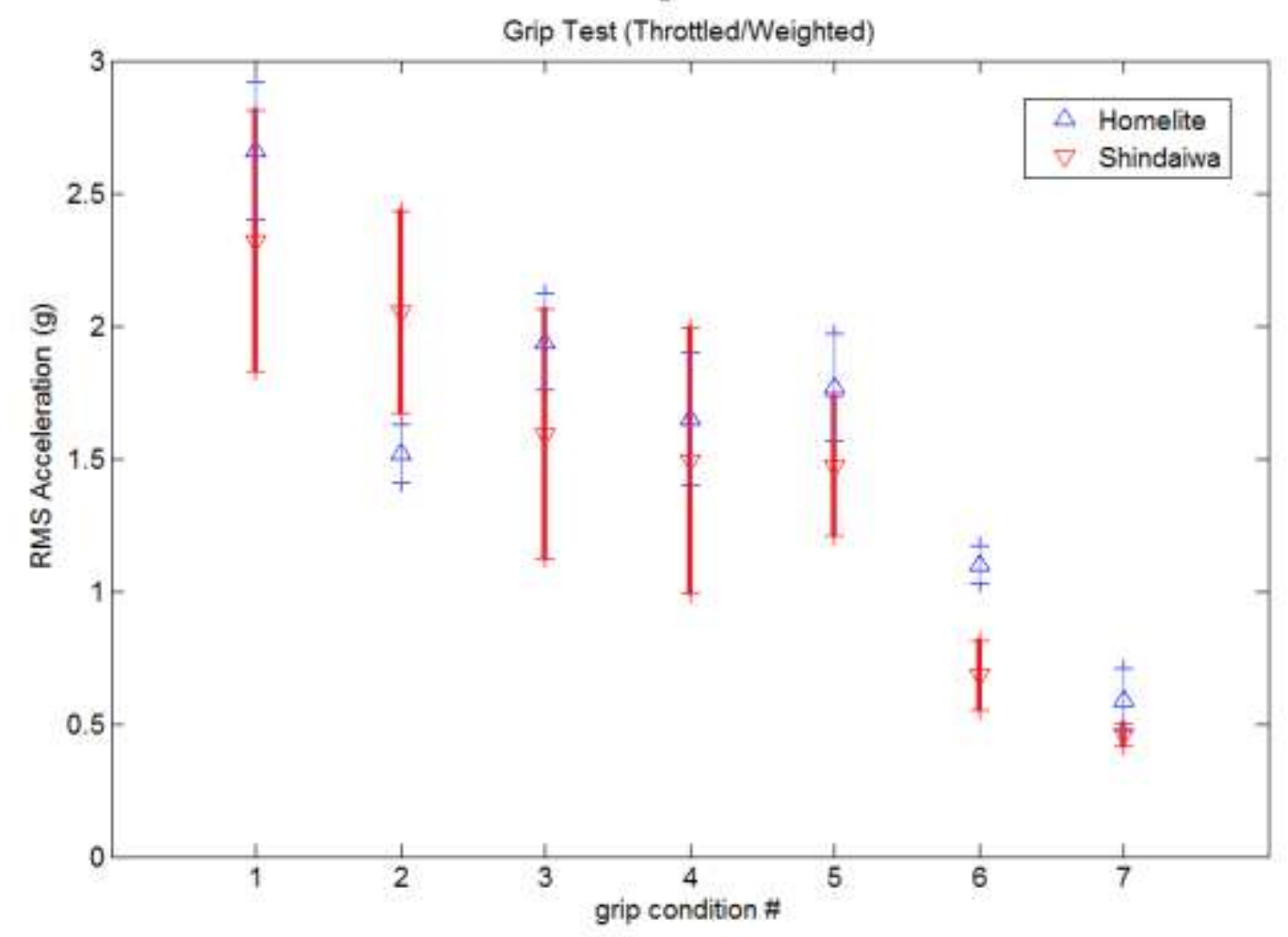

Fig. 12 Performance of various grips under throttled engine conditions (weighted) 
- When the data are weighted to only consider the transmission of harmful frequencies, the Shindaiwa manufacturer's grip (condition \#2) does not seem to offer additional protection over the bare shaft (condition \#1).

- During idling periods (Figs. 9 and 10), none of the studded grips seem to offer any advantage over the manufacturers' respective grips.

- Based on the throttled, unweighted data (Fig. 11), all of the studded grips (conditions $3-7$ ) perform better than either of the manufacturers' grips by reducing the amount of perceived vibration. However, as mentioned previously, a perceived increase in comfort may not reduce HAVS injury potential.

- When the throttled data are weighted so that only the harmful frequencies are taken into consideration, only the grips fabricated from the more compliant, half-inch studded material seem to be effective at lowering the vibration transmitted to the hand.

\section{Discussion}

The compliant mats with half-inch studs are clearly effective at significantly reducing the dose of harmful, lowfrequency vibrations transmitted to a string-trimmer operator's hand and arm in both the Homelite and Shindaiwa units evaluated herein. However, neither the material parameters not the stud geometries were optimized, and parametric studies are underway that specifically evaluate the effects of stud length, areal density, and material parameters on vibration transmission. All of the studded materials used for this study were purchased at a wholesale restaurant supply shop, hence exact material parameters can only be obtained through careful testing. One approach that we are currently using to characterize these materials is modal testing using a frequency- and amplitude-controlled shaker.

Other factors to be considered when developing vibration-reduction approaches for string trimmers include the following:

- While the more compliant half-inch studded mats seemed to be most effective at reducing transmitted vibration, the resulting grip diameter was more awkward to hold and maneuver than the manufacturers' grips. Installing a smaller diameter shaft prior to incorporating the studded wrap may allow the development of an effective anti-vibration grip whose overall diameter is not larger than the diameter of the original manufacturers' grip.

- As part of this work, extensive measurements of the vibrations transmitted through the loop handles of each respective string trimmer were performed. Although not reported here, we found some correlation between transmitted vibration and the tightness of the handle/trimmer shaft interface. We believe that in addition to monitoring screw tightness (as an indication of handle-shaft contact force), studded materials similar to those used for the grip can be incorporated between the handle sleeve and the string trimmer shaft in order to further reduce transmitted vibration. However, in order to accommodate such a modification, either the diameter of the trimmer shaft will need to be reduced, or the handle sleeve will need to be modified to accommodate the larger diameter presented by the wrapped shaft.

- Just as the studded materials proved effective when incorporated as wraps at the engine-side grip location, it is likely that such materials will be similarly effective when incorporated as loop-handle wraps. Experiments to confirm this are currently under way.

\section{Conclusions}

Based on the work reported herein, the following results can be reported:

- Regardless of the support configuration (free-free or held at the two grip locations), both the commercialand consumer-oriented string trimmers produce high-amplitude vibrations. A significant portion of the vibration is produced at the low frequencies considered to be most harmful.

- We have shown that studded materials incorporating both damping and compliant elements can reduce the amplitude of transmitted vibrations at the engine-side grip of string trimmers, including the lowfrequency components.

- The effectiveness of the grip material seems to depend on compliance (vibration attenuation increases with compliance) and stud length (vibration attenuation increases with stud length). Parametric studies are currently under way that will confirm these preliminary findings. 


\section{Acknowledgements}

The authors are grateful for support from Trinity University's Howard Hughes Medical Institute (HHMI) program, as well as the Trinity University Office of Academic Affairs. We also appreciate the participation of Goodwill Industries of San Antonio, TX.

\section{References}

[1] Pelmear, P.L., and Wasserman, D.E., Hand-Arm Vibration, 2nd ed. Beverly Farms, MA: OEM Press, 1998.

[2] Mechanical Vibration - Measurement and Evaluation of Human Exposure to Hand-Transmitted Vibration. ISO 5349, 2001.

[3] Guide for the Measurement and Evaluation of Human Exposure to Vibration Transmitted to the Hand. ANSI S2.70-2006.

[4] Yeo, D. R., http://en.wikipedia.org/wiki/File:Man_weedeating.jpg, accessed 11 OCT 2010.

[5] Shindaiwa Owner's/Operator's Manual. http://www.shindaiwa.com/_docs/oms/trimmers/t272.pdf, accessed 13 JUL 2010.

[6] Homelite Operator's Manual: 26cc Trimmers. Homelite Consumer Products, Inc., Anderson, SC, 19 OCT 2009.

[7] Ko Ying Hao, Investigation of Elastomeric Pad Attenuation of Hand-Transmitted Vibration, MS Thesis, Universiti Sains Malaysia, 2008.

[8] EU Guide to Good Practice on Hand-Arm Vibration, http://www.fosterohs.com/links.htm, accessed 25 OCT 2010.

[9] Andersson, E.R., "Design and Testing of a Vibration Attenuating Handle," International Journal of Industrial Ergonomics, vol 6, 1990, pp. 119-125. 